\title{
Effect of Panchagavya, Jeevamrutha and Cow Urine on Beneficial Microorganisms and Yield of Capsicum (Capsicum annuum L. var. grossum)
}

\author{
B. Boraiah $^{1 *}$, N. Devakumar ${ }^{2}$, S. Shubha ${ }^{3}$ and K.B. Palanna ${ }^{4}$ \\ ${ }^{1}$ Agronomist, AICRP (SM), PC unit, University of Agricultural Sciences, GKVK, Bengaluru, \\ Karnataka, India \\ ${ }^{2}$ College of Agriculture, Hassan, UAS, Bengaluru, Karnataka, India \\ ${ }^{3}$ Microbiology, UAS, Dharwad, Karnataka, India \\ ${ }^{4}$ Plant Pathologist, ARS, Konehalli, UAS, Bengaluru, Karnataka, India \\ *Corresponding author
}

\begin{tabular}{|c|c|}
\hline & A B S T R A C T \\
\hline & $\begin{array}{l}\text { A field experiment was conducted to evaluate the effect of organic liquid formulations on } \\
\text { growth and yield of capsicum at Agricultural Research Station, Arsikere, Karnataka. India. }\end{array}$ \\
\hline Keywords & $\begin{array}{l}\text { The experiment consisting } 12 \text { treatment combinations with three factors viz., Jeevamrutha } \\
(2 \text { levels), Cow urine }(2 \text { levels) and Panchagavya ( } 3 \text { levels). Among different organic }\end{array}$ \\
\hline $\begin{array}{l}\text { Liquid formulation, } \\
\text { Capcimum, } \\
\text { Panchagavya, } \\
\text { Jeevamrutha, Cow } \\
\text { urine, N-fixers, P- } \\
\text { solubilizer. }\end{array}$ & $\begin{array}{l}\text { liquid formulations, application of jeevamrutha recorded significantly higher fruit yield } \\
\left(32.26,39.55,51.63,121.20,100.28,86.40,50.05 \mathrm{q} \mathrm{ha}^{-1} \text { at } 60,70,80,90,100,110 \text { and }\right. \\
120 \mathrm{DAT} \text {, respectively), N-fixers }\left(23.86,24.49 \text { at } 60 \mathrm{DAT} \text { and } 16.79,17.37 \mathrm{X} 10^{3} \text { at }\right. \\
\text { harvest during kharif and summer, respectively) and P-solubilizer }(27.90,31.50 \text { at } 60 \mathrm{DAT} \\
\text { and } 26.68,30.43 \mathrm{X} 10^{3} \text { at harvest during kharif and summer respectively). Significantly } \\
\text { higher fruit yield }\left(30.76,38.0,48.52,117.73,97.15,84.33,48.44 \mathrm{q} \mathrm{h}^{-1} \text { at } 60,70,80,90 \text {, }\right.\end{array}$ \\
\hline Article Info & 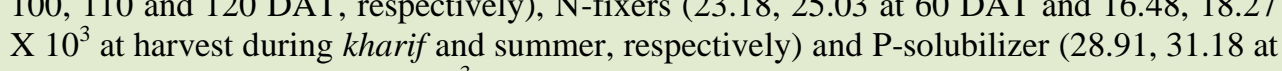 \\
\hline $\begin{array}{l}\text { Accepted: } \\
28 \text { August } 2017 \\
\text { Available Online: } \\
\text { 10 September } 2017\end{array}$ & $\begin{array}{l}60 \mathrm{DAT} \text { and } 27.26,30.34 \times 10^{3} \text { at harvest during kharif and summer, respectively) were } \\
\text { recorded with the application of cow urine. Panchagavya } 6 \text { per cent spray recorded } \\
\text { significantly higher fruit yield }\left(30.25,37.49,48.91,118.91,96.15,86.29,47.81 \mathrm{q} \mathrm{ha}^{-1} \text { at }\right. \\
60,70,80,90,100,110 \text { and } 120 \mathrm{DAT} \text {, respectively), N-fixers life }(23.68,25.59 \text { at } 60 \text { DAT }\end{array}$ \\
\hline & $\begin{array}{l}\text { and } 17.77,17.18 \times 10 \text { at harvest during kharif and summer, respectively) and P- } \\
\text { solubilizer (28.43, } 33.04 \text { at } 60 \mathrm{DAT} \text { and } 27.46,34.53 \times 10^{3} \text { at harvest during kharif and } \\
\text { summer, respectively). }\end{array}$ \\
\hline
\end{tabular}

\section{Introduction}

Organic farming is a holistic way of farming with the aim of conserving the natural resources. Indian agriculture has a better chance to convert itself as organic agriculture because, the per capita and per ha consumption of chemical fertilizers and pesticides in the country is much lower than the global estimates.
Organically produced fruits, vegetables, spices and condiments, crops, medicinal and aromatic plants etc have good keeping quality than that of conventionally grown products. Sustainable agriculture practices can effectively prevent the entry of pesticides and toxicants in the food chain and prevent soil and water pollution. It is adopted with a blend 
of ecologically safe modern technologies. The organic agriculture, though not in its orthodox version, has the potential to be accepted by the farmers (Sreenivasa et al., 2009 and Natarajan, 2007).

Panchagavya, Jeevamruth and Beejamruth are cheaper ecofriendly organic preparations made by cow products namely dung, urine, milk, curd and ghee. The Panchagavya is an efficient plant growth stimulant that enhances the biological efficiency of crops. It is used to activate soil and to protect the plants from diseases and also increase the nutritional quality of fruits and vegetables. It is used as a foliar spray, as soil application along with irrigation water, seed or seedling treatment etc. Three per cent Panchagavya is an ideal concentration for the foliar spray. Jeevamruth promotes immense biological activity in soil and makes the nutrients available to crop. Beejamruth protect the crop from soil borne and seed borne pathogens and it improves seed germination also. (Devakumar et al., 2008) cow urine has got anti-fungal properties and also good source of plant nutrients. It is being used in crop production since ages.

The cost of inorganic fertilizers is increasing enormously to an extent that they are out of reach of small and marginal farmers. Use of inorganic fertilizers and insecticides, the population of beneficial organisms decrease and natural regeneration of nutrition in the soil cease. Soil becomes barren and soil fertility decreases.

The use of fermented liquid manures in such situation is, therefore practically a paying proposal. In these liquid manures, beneficial organisms survive and are helpful in phosphate solubilization, nitrogen fixation etc. Application of these organic liquid formulations will enhance the soil microbial activity and population to a larger extent. This inturn has a positive effect on growth and yield of crops.

\section{Materials and Methods}

A field experiment was conducted at Agricultural Research Station, Arsikere, University of Agricultural Sciences, Bangalore, Karnataka, India. Soil of the experimental plot is red sandy loam, grouped under the classification of Alfisols. Soil is neutral to slight acidic in reaction $\mathrm{pH}(6.42)$, low organic carbon $(0.40 \%)$ and medium in available nitrogen $\left(241.50 \mathrm{~kg} \mathrm{ha}^{-1}\right)$, low available phosphorus $\left(8.80 \mathrm{~kg} \mathrm{ha}^{-1}\right)$ and potassium $\left(231.00 \mathrm{~kg} \mathrm{ha}^{-1}\right)$ content. The trial was laid out on Factorial Randomized Complete Block design with three replications. There were 12 treatment combinations consisting of three factors and they were jeevamrutha (2 levels) - with jeevamrutha $\left(\mathrm{J}_{1}\right)$ and without jeevamrutha $\left(\mathrm{J}_{0}\right)$, cow urine (2 levels) - with cow urine $\left(\mathrm{C}_{1}\right)$ and without cow urine $\left(\mathrm{C}_{0}\right)$ and panchagavya (3) - without panchagavya spray $\left(\mathrm{P}_{0}\right), 3$ per cent panchagavya spray $\left(\mathrm{P}_{1}\right)$ and 6 per cent panchagavya spray $\left(\mathrm{P}_{2}\right)$. Well decomposed farm yard manure $(100 \% \mathrm{~N}$ equivalent basis) was applied 3 weeks before transplanting of capsicum seedlings and incorporated into the soil.

Jeevamrutha (500 litre ha ${ }^{-1}$ ) was applied to the base of the seedlings manually at 25, 50, 75 and 100 DAT, panchagavya was sprayed on 25, 50, 75 and 100 DAT. Diluted mixture of cow urine (2500 litre ha ${ }^{-1}$ ) was applied to the base of the seedlings at vegetative and flowering stages. All cultural operations were carried out as per package of practice. Yield observations were recorded at 60, 70, 80, 90, 100, 110 and 120 DAT.

Before start of the experiment, composite soil samples were drawn from the experimental plot from the upper $0-15 \mathrm{~cm}$ soil layer. The soil was analyzed for biological properties viz., N-fixers and P-solubilizers. Initial microbial count viz., P-solubilizers $(16.32 \mathrm{x}$ $10^{3} \mathrm{cfu} \mathrm{g}^{-1}$ soil $)$ and $\mathrm{N}$-fixers $\left(12 \times 10^{3} \mathrm{cfu} \mathrm{g}^{-1}\right.$ 
soil). Soil samples were collected from the rhizosphere of plants for counting microbial load at 60 DAT and at harvest. Ten gram of soil was serially diluted upto $10^{-6}$ by using sterilized distilled water and cell count per gram of rhizosphere soil was enumerated for P-solublizers $\left(10^{3}\right)$ and free living $\mathrm{N}$-fixer $\left(10^{3}\right)$ by Pikovaskaya ${ }^{e e}$ s media (Himedia) and Waksman No.77 (Aneja, 2003) respectively by following serial dilution plate count technique.

\section{Results and Discussion}

\section{Yield of capsicum $\left(\mathbf{q ~ h a} \mathbf{~}^{-1}\right)$}

Yield per hectare of capsicum at different phonological stages differed significantly due to application of liquid organic formulations (Tables 1a AND 1b). Fruits yield per hectare varied significantly due to the application of jeevamrutha.

Higher yield per hectare was recorded with jeevamrutha $(32.26,39.55,51.63,121.20$, 100.28, 86.40, 50.05 $\mathrm{q} \mathrm{ha}^{-1}$ at 60, 70, 80, 90, 100, 110 and 120 DAT, respectively) while, lower number of plants were observed in without jeevamrutha $(26.54,32.50,38.47$, $104.16,84.48,76.67,41.62 \mathrm{q} \mathrm{ha}^{-1}$ at 60,70 , 80, 90, 100, 110 and 120 DAT, respectively) application.

The beneficial effects of Jeevamrut reported by Palekar (2006), Vasanthkumar (2006) and Devakumar et al., (2008) was attributed to higher microbial load and growth harmones which might have enhanced the soil biomass thereby sustaining the availability and uptake of applied as well as native soil nutrients which ultimately resulted in better growth and yield of crops. Significant differences in yield of capsicum per hectare were observed with application of cow urine. Maximum yield of capsicum per hectare were observed with application of cow urine $(30.76,38.0,48.52$, $117.73,97.15,84.33,48.44 \mathrm{q} \mathrm{ha}^{-1}$ at 60,70 ,
80, 90, 100, 110 and 120 DAT, respectively) whereas, minimum yield of capsicum per hectare were observed in without cow urine (28.05, 34.05, 41.58, 107.62, 87.61, 78.54, $43.24 \mathrm{q} \mathrm{ha}^{-1}$ at 60, 70, 80, 90, 100, 110 and 120 DAT, respectively). This is in conformity with Reddy et al., (2010) who have reported higher yield levels obtained with application of biodigester liquid manures to many field crops. Similarly, Siddaram, (2012) have also reported increased yield levels of rice with biodigester liquid manures. Panchagavya spray influenced significantly on yield of capsicum per hectare.

Spraying of $6 \%$ panchagavya recorded capsicum yield per hectare of $30.25,37.49$, $48.91,118.91,96.15,86.29,47.81 \mathrm{q} \mathrm{ha}^{-1}$ at 60, 70, 80, 90, 100, 110 and 120 DAT, respectively and lower capsicum yield per hectare of 28.41, 34.51, 41.78, 106.20, 88.91, $77.11,43.82$ at 60, 70, 80, 90, 100, 110 and 120 DAT, respectively were noticed in without panchagavya spray. This might be due to adequate supply of nutrients at different growth stages of the crop as well as presence of growth regulators in Panchagavya contributing to higher yield (Sridhar et al., 2001 and Somasundaram et al., 2003 and Natarajan, 2007).

The yield of any crop plants depends on the assimilatory surface of the plant system. A sound source in terms of plant height, LAI, number of branches to support and the leaves are logically able to increase the dry matter and its distribution in different parts is important for determination of total yield of the crop (Krishnamurthy, 2012). Number of fruits per plant did not differ significantly due to the interaction effect of jeevamrutha and cow urine, jeevamrutha and panchagavya and cow urine and panchagavya. Yield per hectare of capsicum did not vary significantly due to the interaction effect of jeevamrutha and cow urine, jeevamrutha and panchagavya and cow urine and panchagavya. 
Table.1a Effect of Panchagavya, Jeevamrutha and cow urine application on fruit yield per hectare (q) of capsicum pooled data of two seasons

\begin{tabular}{|c|c|c|c|c|c|c|c|c|c|c|c|c|}
\hline \multirow{4}{*}{ Organic liquid formulations } & \multicolumn{12}{|c|}{ Fruit yield per hectare (q) } \\
\hline & \multicolumn{3}{|c|}{60 DAT } & \multicolumn{3}{|c|}{70 DAT } & \multicolumn{3}{|c|}{80 DAT } & \multicolumn{3}{|c|}{90 DAT } \\
\hline & \multicolumn{12}{|c|}{ Jeevamrutha (J) } \\
\hline & $\begin{array}{l}\text { without } \\
\left(\mathrm{J}_{0}\right)\end{array}$ & $\begin{array}{c}\text { with } \\
\left(\mathrm{J}_{1}\right) \\
\end{array}$ & Mean & $\begin{array}{c}\text { without } \\
\left(\mathrm{J}_{0}\right)\end{array}$ & $\begin{array}{l}\text { with } \\
\left(\mathrm{J}_{1}\right)\end{array}$ & Mean & $\begin{array}{c}\text { without } \\
\left(\mathrm{J}_{0}\right)\end{array}$ & $\begin{array}{l}\text { with } \\
\left(\mathrm{J}_{1}\right)\end{array}$ & Mean & $\begin{array}{c}\text { without } \\
\left(\mathrm{J}_{0}\right)\end{array}$ & $\begin{array}{l}\text { with } \\
\left(\mathrm{J}_{1}\right)\end{array}$ & Mean \\
\hline Cow urine $(\mathrm{C})$ & & & & & & & & & & & & \\
\hline without & 25.19 & 30.90 & 28.05 & 30.21 & 37.90 & 34.05 & 36.35 & 46.82 & 41.58 & 100.75 & 114.49 & 107.62 \\
\hline with & 27.90 & 33.62 & 30.76 & 34.79 & 41.21 & 38.00 & 40.60 & 56.44 & 48.52 & 107.56 & 127.91 & 117.73 \\
\hline \multirow[t]{2}{*}{ Mean } & 26.54 & 32.26 & & 32.50 & 39.55 & & 38.47 & 51.63 & & 104.16 & 121.20 & \\
\hline & S.Em \pm & C.D. & & S.Em \pm & C.D. & & S.Em \pm & C.D. & & S.Em \pm & C.D. & \\
\hline \multirow{2}{*}{$\begin{array}{l}\text { Jeevamrutha }(\mathrm{J}) \\
\text { Cow urine }(\mathrm{C})\end{array}$} & 0.25 & 0.75 & & 0.51 & 1.50 & & 0.86 & 2.52 & & 1.80 & 5.27 & \\
\hline & 0.25 & 0.75 & & 0.51 & 1.50 & & 0.86 & 2.52 & & 1.80 & 5.27 & \\
\hline $\begin{array}{c}\text { Cow urine (C) } \\
\mathrm{J} \times \mathrm{C}\end{array}$ & 0.36 & NS & & 0.72 & NS & & 1.22 & 3.57 & & 2.54 & NS & \\
\hline Panchagavya spray (P) & & & & & & & & & & & & \\
\hline $\mathrm{P}_{0} \quad 0 \%$ & 25.32 & 31.50 & 28.41 & 30.97 & 38.04 & 34.51 & 36.72 & 46.85 & 41.78 & 98.94 & 113.45 & 106.20 \\
\hline $3 \%$ & 26.66 & 32.23 & 29.45 & 32.49 & 39.67 & 36.08 & 38.23 & 50.68 & 44.45 & 104.79 & 121.06 & 112.92 \\
\hline $6 \%$ & 27.65 & 33.05 & 30.35 & 34.04 & 40.94 & 37.49 & 40.46 & 57.37 & 48.91 & 108.74 & 129.09 & 118.91 \\
\hline \multirow{2}{*}{ Mean } & 26.54 & 32.26 & & 32.50 & 39.55 & & 38.47 & 51.63 & & 104.16 & 121.20 & \\
\hline & S.Em \pm & C.D. & & S.Em \pm & C.D. & & S.Em \pm & C.D. & & S.Em \pm & C.D. & \\
\hline \multirow{4}{*}{$\begin{array}{c}\text { Panchagavya spray (P) } \\
\text { J x P }\end{array}$} & 0.31 & 0.91 & & 0.63 & 1.84 & & 1.05 & 3.09 & & 2.20 & 6.46 & \\
\hline & 0.44 & NS & & 0.89 & NS & & 1.49 & NS & & 3.11 & NS & \\
\hline & \multicolumn{12}{|c|}{ Panchagavya spray (P) } \\
\hline & $\mathrm{P}_{0}$ & $\mathrm{P}_{1}$ & $\mathrm{P}_{2}$ & $\mathrm{P}_{0}$ & $\mathrm{P}_{1}$ & $\mathrm{P}_{2}$ & $\mathrm{P}_{0}$ & $\mathrm{P}_{1}$ & $\mathrm{P}_{2}$ & $\mathrm{P}_{0}$ & $\mathrm{P}_{1}$ & $\mathrm{P}_{2}$ \\
\hline Cow urine $(C)$ & & & & & & & & & & & & \\
\hline without & 27.12 & 27.97 & 29.05 & 33.20 & 34.04 & 34.92 & 38.87 & 41.01 & 44.86 & 101.47 & 108.08 & 113.32 \\
\hline with & 29.70 & 30.93 & 31.64 & 35.81 & 38.12 & 40.07 & 44.70 & 47.89 & 52.97 & 110.93 & 117.76 & 124.51 \\
\hline \multirow{2}{*}{$\mathrm{C} \times \mathrm{P}$} & S.Em \pm & C.D. & & S.Em \pm & C.D. & & S.Em \pm & C.D. & & S.Em \pm & C.D. & \\
\hline & 0.44 & NS & & 0.89 & NS & & 1.49 & NS & & 3.11 & NS & \\
\hline
\end{tabular}


Table.1b Effect of Panchagavya, Jeevamrutha and cow urine application on fruit yield per hectare (q) of capsicum pooled data of two seasons

\begin{tabular}{|c|c|c|c|c|c|c|c|c|c|c|c|c|}
\hline \multirow{4}{*}{ Organic liquid formulations } & \multicolumn{12}{|c|}{ Fruit yield per hectare (q) } \\
\hline & \multicolumn{3}{|c|}{100 DAT } & \multicolumn{3}{|c|}{110 DAT } & \multicolumn{3}{|c|}{120 DAT } & \multicolumn{3}{|c|}{ Cumulative } \\
\hline & \multicolumn{12}{|c|}{ Jeevamrutha $(\mathbf{J})$} \\
\hline & $\begin{array}{c}\text { without } \\
\left(\mathrm{J}_{0}\right)\end{array}$ & $\begin{array}{c}\text { with } \\
\left(\mathrm{J}_{1}\right)\end{array}$ & Mean & $\begin{array}{c}\text { without } \\
\left(\mathrm{J}_{0}\right)\end{array}$ & $\begin{array}{l}\text { with } \\
\left(\mathrm{J}_{1}\right)\end{array}$ & Mean & $\begin{array}{c}\text { without } \\
\left(\mathrm{J}_{0}\right)\end{array}$ & $\begin{array}{c}\text { with } \\
\left(\mathrm{J}_{1}\right)\end{array}$ & Mean & $\begin{array}{c}\text { without } \\
\left(\mathrm{J}_{0}\right)\end{array}$ & $\begin{array}{c}\text { with } \\
\left(\mathrm{J}_{1}\right)\end{array}$ & Mean \\
\hline Cow urine $(\mathrm{C})$ & & & & & & & & & & & & \\
\hline without & 78.83 & 96.39 & 87.61 & 73.75 & 83.32 & 78.54 & 38.37 & 48.10 & 43.24 & 383.45 & 457.91 & 420.68 \\
\hline with & 90.13 & 104.18 & 97.15 & 79.58 & 89.48 & 84.53 & 44.87 & 52.00 & 48.44 & 425.42 & 504.84 & 465.13 \\
\hline \multirow{2}{*}{ Mean } & 84.48 & 100.28 & & 76.67 & 86.40 & & 41.62 & 50.05 & & 404.44 & 481.37 & \\
\hline & S.Em \pm & C.D. & & S.Em \pm & C.D. & & S.Em \pm & C.D. & & S.Em \pm & C.D. & \\
\hline Jeevamrutha $(\mathrm{J})$ & 1.19 & 3.48 & & 1.58 & 4.63 & & 0.60 & 1.76 & & 4.94 & 14.49 & \\
\hline Cow urine $(\mathrm{C})$ & 1.19 & 3.48 & & 1.58 & 4.63 & & 0.60 & 1.76 & & 4.94 & 14.49 & \\
\hline $\mathrm{J} \times \mathrm{C}$ & 1.68 & NS & & 2.23 & NS & & 0.85 & NS & & 6.99 & NS & \\
\hline Panchagavya spray (P) & & & & & & & & & & & & \\
\hline $0 \%$ & 79.83 & 97.99 & 88.91 & 72.20 & 82.03 & 77.11 & 39.72 & 47.92 & 43.82 & 383.70 & 457.78 & 420.74 \\
\hline $3 \%$ & 84.68 & 99.48 & 92.08 & 76.57 & 85.81 & 81.19 & 42.06 & 49.70 & 45.88 & 405.48 & 478.63 & 442.05 \\
\hline $6 \%$ & 88.93 & 103.37 & 96.15 & 81.23 & 91.36 & 86.29 & 43.09 & 52.53 & 47.81 & 424.14 & 507.71 & 465.92 \\
\hline \multirow[t]{2}{*}{ Mean } & 84.48 & 100.28 & & 76.67 & 86.40 & & 41.62 & 50.05 & & 404.44 & 481.37 & \\
\hline & S.Em \pm & C.D. & & S.Em \pm & C.D. & & S.Em \pm & C.D. & & S.Em \pm & C.D. & \\
\hline \multirow{4}{*}{$\begin{array}{c}\text { Panchagavya spray (P) } \\
\text { J x P }\end{array}$} & 1.45 & 4.26 & & 1.93 & 5.67 & & 0.74 & 2.16 & & 6.05 & 17.75 & \\
\hline & 2.06 & NS & & 2.74 & NS & & 1.04 & NS & & 8.56 & NS & \\
\hline & \multicolumn{12}{|c|}{ Panchagavya spray (P) } \\
\hline & $\mathrm{P}_{0}$ & $\mathrm{P}_{1}$ & $\mathrm{P}_{2}$ & $\mathrm{P}_{0}$ & $\mathrm{P}_{1}$ & $\mathrm{P}_{2}$ & $\mathrm{P}_{0}$ & $\mathrm{P}_{1}$ & $\mathrm{P}_{2}$ & $\mathrm{P}_{0}$ & $\mathrm{P}_{1}$ & $\mathrm{P}_{2}$ \\
\hline Cow urine $(C)$ & & & & & & & & & & & & \\
\hline without & 84.30 & 87.53 & 91.00 & 75.44 & 78.52 & 81.65 & 41.01 & 43.51 & 45.19 & 401.41 & 420.67 & 439.98 \\
\hline with & 93.52 & 96.63 & 101.31 & 78.79 & 83.86 & 90.94 & 46.63 & 48.25 & 50.43 & 440.07 & 463.44 & 491.87 \\
\hline \multirow{2}{*}{$\mathrm{C} \times \mathrm{P}$} & S.Em \pm & C.D. & & S.Em \pm & C.D. & & S.Em \pm & C.D. & & S.Em \pm & C.D. & \\
\hline & 2.06 & NS & & 2.74 & NS & & 1.04 & NS & & 8.56 & NS & \\
\hline
\end{tabular}


Table.2 Effect of Panchagavya, Jeevamrutha and cow urine application on N-fixing microorganisms at 60 DAT and after harvesting of capsicum

\begin{tabular}{|c|c|c|c|c|c|c|c|c|c|c|c|c|}
\hline \multirow{5}{*}{ Organic liquid formulations } & \multicolumn{12}{|c|}{$\mathrm{N}$-fixers (No. $10^{3} \times \mathrm{cfu} \mathrm{g}^{-1}$ soil) } \\
\hline & \multicolumn{6}{|c|}{ Kharif } & \multicolumn{6}{|c|}{ Summer } \\
\hline & \multicolumn{3}{|c|}{ 60 DAT } & \multicolumn{3}{|c|}{ After harvesting } & \multicolumn{3}{|c|}{60 DAT } & \multicolumn{3}{|c|}{ After harvesting } \\
\hline & \multicolumn{12}{|c|}{ Jeevamrutha $(\mathrm{J})$} \\
\hline & $\begin{array}{c}\text { without } \\
\left(\mathrm{J}_{0}\right)\end{array}$ & $\begin{array}{l}\text { with } \\
\left(\mathrm{J}_{1}\right)\end{array}$ & Mean & $\begin{array}{c}\text { without } \\
\left(\mathrm{J}_{0}\right)\end{array}$ & $\begin{array}{l}\text { with } \\
\left(\mathrm{J}_{1}\right)\end{array}$ & Mean & $\begin{array}{c}\text { without } \\
\left(\mathrm{J}_{0}\right)\end{array}$ & $\begin{array}{l}\text { with } \\
\left(\mathrm{J}_{1}\right)\end{array}$ & Mean & $\begin{array}{c}\text { without } \\
\left(\mathrm{J}_{0}\right)\end{array}$ & $\begin{array}{l}\text { with } \\
\left(\mathrm{J}_{1}\right)\end{array}$ & Mean \\
\hline Cow urine $(\mathrm{C})$ & & & & & & & & & & & & \\
\hline without & 19.75 & 22.92 & 21.33 & 14.04 & 16.52 & 15.28 & 23.71 & 23.96 & 23.83 & 14.72 & 14.73 & 14.73 \\
\hline with & 21.56 & 24.81 & 23.18 & 15.91 & 17.06 & 16.48 & 25.03 & 25.03 & 25.03 & 16.54 & 20.01 & 18.27 \\
\hline \multirow[t]{2}{*}{ Mean } & 20.65 & 23.86 & & 14.97 & 16.79 & & 24.37 & 24.49 & & 15.63 & 17.37 & \\
\hline & S.Em \pm & C.D. & & S.Em \pm & C.D. & & S.Em \pm & C.D. & & S.Em \pm & C.D. & \\
\hline Jeevamrutha $(\mathrm{J})$ & 0.78 & 2.30 & & 0.68 & NS & & 0.90 & NS & & 0.86 & NS & \\
\hline Cow urine $(\mathrm{C})$ & 0.78 & NS & & 0.68 & NS & & 0.90 & NS & & 0.86 & 2.52 & \\
\hline $\mathrm{J} \times \mathrm{C}$ & 1.11 & NS & & 0.96 & NS & & 1.28 & NS & & 1.22 & NS & \\
\hline Panchagavya spray (P) & & & & & & & & & & & & \\
\hline $0 \%$ & 19.27 & 21.90 & 20.59 & 13.71 & 15.70 & 14.70 & 22.58 & 22.33 & 22.46 & 14.09 & 16.89 & 15.49 \\
\hline $3 \%$ & 21.56 & 25.79 & 22.51 & 14.36 & 15.98 & 15.17 & 25.12 & 26.07 & 25.24 & 16.14 & 17.52 & 16.83 \\
\hline $6 \%$ & 21.13 & 23.89 & 23.68 & 16.86 & 18.68 & 17.77 & 25.40 & 25.08 & 25.59 & 16.66 & 17.70 & 17.18 \\
\hline \multirow[t]{2}{*}{ Mean } & 20.65 & 23.86 & & 14.97 & 16.79 & & 24.37 & 24.49 & & 15.63 & 17.37 & \\
\hline & S.Em \pm & C.D. & & S.Em \pm & C.D. & & S.Em \pm & C.D. & & S.Em \pm & C.D. & \\
\hline \multirow{4}{*}{$\begin{array}{c}\text { Panchagavya spray }(\mathrm{P}) \\
\text { J X P }\end{array}$} & 0.96 & NS & & 0.83 & 2.44 & & 1.11 & NS & & 1.05 & NS & \\
\hline & 1.36 & NS & & 1.18 & NS & & 1.56 & NS & & 1.49 & NS & \\
\hline & \multicolumn{12}{|c|}{ Panchagavya spray $(\mathbf{P})$} \\
\hline & $\mathrm{P}_{0}$ & $\mathrm{P}_{1}$ & $\mathrm{P}_{2}$ & $\mathrm{P}_{0}$ & $\mathrm{P}_{1}$ & $\mathrm{P}_{2}$ & $\mathrm{P}_{0}$ & $\mathrm{P}_{1}$ & $\mathrm{P}_{2}$ & $\mathrm{P}_{0}$ & $\mathrm{P}_{1}$ & $\overline{\mathrm{P}_{2}}$ \\
\hline Cow urine $(\mathrm{C})$ & & & & & & & & & & & & \\
\hline without & 18.39 & 23.43 & 22.18 & 13.01 & 15.35 & 17.47 & 20.95 & 25.41 & 25.15 & 13.12 & 17.17 & 13.89 \\
\hline with & 22.78 & 23.92 & 22.84 & 16.40 & 14.99 & 18.06 & 23.97 & 25.78 & 25.34 & 17.86 & 16.49 & 20.47 \\
\hline \multirow{2}{*}{$\mathrm{C} \times \mathrm{P}$} & S.Em \pm & C.D. & & S.Em \pm & C.D. & & S.Em \pm & C.D. & & S.Em \pm & C.D. & \\
\hline & 1.36 & NS & & 1.18 & NS & & 1.56 & NS & & 1.49 & NS & \\
\hline
\end{tabular}


Table.3 Effect of Panchagavya, Jeevamrutha and cow urine application on P-solubilizing microorganisms at 60 DAT and after harvesting of capsicum

\begin{tabular}{|c|c|c|c|c|c|c|c|c|c|c|c|c|}
\hline \multirow{5}{*}{ Organic liquid formulations } & \multicolumn{12}{|c|}{ P-solubilizers (No. $10^{3} \times \mathrm{cfu} \mathrm{g}^{-1}$ soil) } \\
\hline & \multicolumn{6}{|c|}{ Kharif } & \multicolumn{6}{|c|}{ Summer } \\
\hline & \multicolumn{3}{|c|}{ 60 DAT } & \multicolumn{3}{|c|}{ After harvesting } & \multicolumn{3}{|c|}{60 DAT } & \multicolumn{3}{|c|}{ After harvesting } \\
\hline & \multicolumn{12}{|c|}{ Jeevamrutha $(\mathbf{J})$} \\
\hline & $\begin{array}{c}\text { without } \\
\left(\mathrm{J}_{0}\right)\end{array}$ & $\begin{array}{c}\text { with } \\
\left(\mathrm{J}_{1}\right)\end{array}$ & Mean & $\begin{array}{c}\text { without } \\
\left(\mathrm{J}_{0}\right)\end{array}$ & $\begin{array}{c}\text { with } \\
\left(\mathrm{J}_{1}\right)\end{array}$ & Mean & $\begin{array}{c}\text { without } \\
\left(\mathrm{J}_{0}\right)\end{array}$ & $\begin{array}{c}\text { with } \\
\left(\mathrm{J}_{1}\right)\end{array}$ & Mean & $\begin{array}{c}\text { without } \\
\left(\mathrm{J}_{0}\right)\end{array}$ & $\begin{array}{c}\text { with } \\
\left(\mathrm{J}_{1}\right)\end{array}$ & Mean \\
\hline Cow urine $(\mathrm{C})$ & & & & & & & & & & & & \\
\hline without & 23.62 & 25.04 & 24.33 & 22.49 & 24.45 & 23.47 & 29.33 & 30.74 & 30.03 & 27.72 & 29.22 & 28.47 \\
\hline with & 27.07 & 30.76 & 28.91 & 25.61 & 28.91 & 27.26 & 30.11 & 32.26 & 31.18 & 29.03 & 31.64 & 30.34 \\
\hline \multirow{2}{*}{ Mean } & 25.35 & 27.90 & & 24.05 & 26.68 & & 29.72 & 31.50 & & 28.37 & 30.43 & \\
\hline & S.Em \pm & C.D. & & S.Em \pm & C.D. & & S.Em \pm & C.D. & & S.Em \pm & C.D. & \\
\hline Jeevamrutha $(\mathrm{J})$ & 0.40 & 1.17 & & 0.44 & 1.29 & & 0.57 & 1.66 & & 0.90 & NS & \\
\hline Cow urine $(\mathrm{C})$ & 0.40 & 1.17 & & 0.44 & 1.29 & & 0.57 & $\mathrm{NS}$ & & 0.90 & NS & \\
\hline $\mathrm{J} \times \mathrm{C}$ & 0.57 & NS & & 0.62 & NS & & 0.80 & NS & & 1.27 & NS & \\
\hline Panchagavya spray (P) & & & & & & & & & & & & \\
\hline $0 \%$ & 24.14 & 26.23 & 25.19 & 22.79 & 24.66 & 23.73 & 26.58 & 29.13 & 27.85 & 22.01 & 24.83 & 23.42 \\
\hline $3 \%$ & 25.00 & 27.50 & 26.25 & 23.95 & 25.85 & 24.90 & 30.01 & 31.86 & 30.93 & 28.89 & 31.63 & 30.26 \\
\hline $6 \%$ & 26.90 & 29.96 & 28.43 & 25.41 & 29.52 & 27.46 & 32.57 & 33.52 & 33.04 & 34.23 & 34.83 & 34.53 \\
\hline \multirow[t]{2}{*}{ Mean } & 25.35 & 27.90 & & 24.05 & 26.68 & & 29.72 & 31.50 & & 28.37 & 30.43 & \\
\hline & S.Em \pm & C.D. & & S.Em \pm & C.D. & & S.Em \pm & C.D. & & S.Em \pm & C.D. & \\
\hline \multirow{4}{*}{$\begin{array}{c}\text { Panchagavya spray (P) } \\
\text { J x P }\end{array}$} & 0.49 & 1.44 & & 0.54 & 1.58 & & 0.69 & 2.04 & & 1.10 & 3.23 & \\
\hline & 0.69 & NS & & 0.76 & NS & & 0.98 & NS & & 1.56 & NS & \\
\hline & \multicolumn{12}{|c|}{ Panchagavya spray (P) } \\
\hline & $\mathrm{P}_{0}$ & $\mathrm{P}_{1}$ & $\mathrm{P}_{2}$ & $\mathrm{P}_{0}$ & $\mathrm{P}_{1}$ & $\mathrm{P}_{2}$ & $\mathrm{P}_{0}$ & $\mathrm{P}_{1}$ & $\mathrm{P}_{2}$ & $\mathrm{P}_{0}$ & $\mathrm{P}_{1}$ & $\mathrm{P}_{2}$ \\
\hline Cow urine $(C)$ & & & & & & & & & & & & \\
\hline without & 22.97 & 24.33 & 25.69 & 22.02 & 23.31 & 25.08 & 27.22 & 30.15 & 32.73 & 20.70 & 30.44 & 34.26 \\
\hline with & 27.41 & 28.17 & 31.17 & 25.44 & 26.49 & 29.85 & 28.48 & 31.72 & 33.35 & 26.14 & 30.07 & 34.80 \\
\hline \multirow{2}{*}{$\mathrm{C} \times \mathrm{P}$} & S.Em \pm & C.D. & & S.Em \pm & C.D. & & S.Em \pm & C.D. & & S.Em \pm & C.D. & \\
\hline & 0.69 & NS & & 0.76 & NS & & 0.98 & NS & & 1.56 & NS & \\
\hline
\end{tabular}




\section{Microbial properties}

\section{Nitrogen fixers (N-fixers) (No. $x 1^{3} \mathrm{cfu}^{-1}$ soil)}

Application of different organic liquid formulations influenced nitrogen fixer's population at different growth stages (Table 2). Significantly higher nitrogen fixers were observed due to the application of jeevamrutha. Higher nitrogen fixers were observed with jeevamrutha $(23.86,24.49$ at 60 DAT and $16.79,17.37$ at harvest during kharif and summer, respectively) and lower was recorded in without jeevamrutha $(20.65,24.37$ at 60 DAT and $14.97,15.63$ at harvest during kharif and summer, respectively). Jeevamruth contains enormous amount of microbial load which multiply in the soil and acts as tonic to enhance microbial activity in soil (Palekar, 2006). There was significant difference in Nitrogen fixers with application of cow urine application. Maximum nitrogen fixers were observed with application of cow urine $(23.18,25.03$ at 60 DAT and 16.48, 18.27 at harvest during kharif and summer, respectively) whereas, minimum was observed in without cow urine (21.33, 23.83 at 60 DAT and 15.28, 14.73 at harvest during kharif and summer, respectively). Panchagavya spray influenced significantly on nitrogen fixers. Spraying of $6 \%$ recorded higher Nitrogen fixers $(23.68,25.59$ at 60 DAT and 17.77, 17.18 at harvest during kharif and summer, respectively) and lower was recorded in without panchagavya spray $(20.59,22.46$ at 60 DAT and 14.70, 15.49 at harvest during kharif and summer, respectively). The organic liquid manures viz., Panchagavya, Beejamruth and Jeevamruth prepared by using cow products are known to contain beneficial microflora like Azospirillum, Azotobacter, phosphobacteria, Pseudomonas, lactic acid bacteria and Methylotrophs in abundant numbers and also contain some useful fungi and actinomyctes (Sreenivasa, 2009 and Palekar, 2006). Nitrogen fixers did not differ significantly due to the interaction effect of jeevamrutha and cow urine, jeevamrutha and panchagavya and cow urine and panchagavya.

\section{P-solubilizers (No. x $10^{3} \mathrm{cfu} \mathrm{g}^{-1}$ soil)}

Phosphorus solubilizers at different growth stages as influenced by different organic liquid formulations (Table 3). Significant difference observed in P-solubilizers due to the application of jeevamrutha. Higher P-solubilizers were observed with jeevamrutha $(27.90,31.50$ at 60 DAT and 26.68, 30.43 at harvest during kharif and summer, respectively) and lower was recorded in without jeevamrutha $(25.35,29.72$ at 60 DAT and 24.05, 28.37 at harvest during kharif and summer, respectively). The results of the study are in accordance with Devakumar et al., (2008). Higher bacteria, fungi, actinomycetes, $\mathrm{N}$ - fixers and $\mathrm{P}$-solubilizers were reported in organic liquid formulations such as jeevamrutha and panchagavya. There was significant difference in P-solubilizers with application of cow urine application. Maximum P-solubilizers were observed with application of cow urine $(28.91,31.18$ at 60 DAT and 27.26, 30.34 at harvest during kharif and summer, respectively) whereas, minimum was observed in without cow urine $(24.33,30.03$ at 60 DAT and $23.47,28.47$ at harvest during kharif and summer, respectively). Yadav and Mowade (2004) opined that this increase might be due to cumulative effect of liquid organic inputs in increasing organic carbon content of soil which acted as carbon and energy source for microbes and in quick buildup of heterotrophic microflora and fauna. Similar results were obtained by Shwetha (2008), who reported the nutrient management through organics in soybean-wheat cropping system and found that the application of organic manures supplemented with fermented organics resulted in the significant improvement of soil microbial population and enzymatic activity. Panchagavya spray influenced significantly on P-solubilizers. Spraying of $6 \%$ recorded higher P-solubilizers (28.43, 33.04 at 60 DAT and 27.46, 34.53 at harvest during kharif and summer, respectively) and lower was recorded in without panchagavya spray $(25.19,27.85$ at 60 DAT and 23.73, 23.42 at harvest during kharif and summer, respectively). The foliar spray of panchagavya 
might have enhanced microbial activity on the plant parts like on leaves, shoot and fruits. Natarajan (2007) reported that the Panchagavya contains macronutrients like $\mathrm{N}, \mathrm{P}$ and $\mathrm{K}$, essential micronutrients, many vitamins, essential amino acids, growth promoting factors like IAA, GA, which may provide nutrition to rhizosphere microorganisms and thus help to increase their population. P-solubilizers did not differ significantly due to the interaction effect of jeevamrutha and cow urine, jeevamrutha and panchagavya and cow urine and panchagavya.

\section{References}

Aneja, K.R., 2003. Experiments in micro biology, plant pathology and biotechnology, IVEd. 157.

Devakumar, N., Rao, G. G. E., Shubha, S., Imrankhan, Nagaraj and Gowda, S. B. 2008. Activities of Organic Farming Research Centre. Navile, Shimoga, Univ. Agri. Sci., Bangalore, Karnataka.

Krishnamurthy, R., 2012. Productivity and economics of rainfed rice as influenced by intergrated nutrient management. Madras Agric. J., 99 (4-6): 266-270.

Natarajan, K., 2007. Panchagavya for plant. Proc. Nation. Conf. on Glory of Gomatha, Dec. 1- 3, S. V. Veterinary Uni, Tirupati, A. P., pp. 72-75.

Palekar, S., 2006. Shoonya bandovalada naisargika krushi pub. Swamy Anand, Agri Prakashana, Bangalore.

Reddy, V. C., Jayaram Reddy, M., Sannathimappa, Byrappanavar, S. T., Girijesh, G. K., Narayanaprasad, Vasanthakumar, H. L., Agadeesh, B. R., Mohan, G. S., Hanumanthappa, H., Panduranga, RAO, G. G. E.
Vijayalakshman, Govindaraju, C., Basavaraja Naik, T. and Pradeep, S., 2010. Breakthrough in organic research. Ann. Prog. Report, Research Institute on Organic Farming. Univ. Agric. Sci., Bangalore. pp. 9-21.

Shwetha, B. N., 2008, Effect of nutrient management through the organics in soybean-wheat cropping system. M. Sc (Agri.) Thesis, Univ. Agric. Sci. Dharwad.

Siddaram, 2012. Effect of FYM and biodigester liquid manure on the performance of aerobic rice - field bean cropping sequence, Ph.D. Thesis, Univ. of Agric. Sci., Bangalore, Karnataka.

Somasundaram, E., Sankaran, N., Meena, S., Thyagarajan, T. M., K. Chandragiri and Pannerselvam. S. 2003. Response of greengram to varied level of panchagavya (organic nutrition) foliar spray. Madras Agric. J., 90: 169-172.

Sreenivasa, M. N., Nagaraj. M., Naik and Bhat, S. N. 2009. Beneficial traits of microbial isolates of organic liquid manures. First Asian PGPR Congress for sustainable agriculture, 21-24 June, ANGRAU, Hyderabad.

Sridhar, S. S., Arunmugasamy, K., Vijaya Lakshmi and Balasubramanian, A.V. 2001. Vrikshayurveda, Ayurveda for plant. A user manual. Clarion, 1-6.

Vasanthkumar, H. H. R., 2006, Jeevamrut slurry preparation. Siri Samruddhi, 4-5.

Yadav, A. K., and Mowade, S. M. 2004. Organic manures and compost. In: Organic Farming. Ray of Hope for Indian Farmer.

\section{How to cite this article:}

Boraiah, B., N. Devakumar, S. Shubha and Palanna, K.B. 2017. Effect of Panchagavya, Jeevamrutha and Cow Urine on Beneficial Microorganisms and Yield of Capsicum (Capsicum annuum L. var. grossum). Int.J.Curr.Microbiol.App.Sci. 6(9): 3226-3234.

doi: https://doi.org/10.20546/ijcmas.2017.609.397 